\title{
Equilibrium model of urban taxi service network based on the integrated
}

\section{service modes}

\author{
ZHU BAI ${ }^{1, a}$, JIAN WANG ${ }^{2, b}$ \\ ${ }^{1}$ School of Transportation Science and Engineering, Harbin Institute of Technology, Harbin, China \\ ${ }^{2}$ School of Transportation Science and Engineering, Harbin Institute of Technology, Harbin, China \\ abaizhu1979@yahoo.com.cn, bwang_jian@hit.edu.cn
}

Keywords: taxi service; network equilibrium; cruising taxi mode; dispatch taxi mode

\begin{abstract}
This paper proposes an equilibrium model of urban taxi services on network to present the impact of the integrated service modes and the influence of the elastic customer demand distribution on taxi network equilibrium. Based on the cruising service mode, the model can describe how occupied taxis and vacant taxis cruise on network to provide services and search for customers by considering both the expected time cost and ride revenue and the elastic distribution characteristic of customer demand. At the same time, the dispatch service equilibrium problem by researching trip mode choice behavior is also considered. The model can then establish the relationship between customer waiting time and taxi search/waiting time by introducing a taxi-customer meeting function. And moreover, in supply-demand equilibrium, a number of performance measures of the taxis market can be obtained, such as the number of cruising taxis, the number of dispatch taxis and the average customer waiting time.
\end{abstract}

\section{Introduction}

As the urbanization speeds up, people have increasingly paid attention to the trip mode of taxi which is regarded as the important supplementary mode of public transit due to a flexible and speedy service offered by taxi. Especially with the frequent fluctuation of gas price, the increasing attention to environmental protection and the constant occurrence of the supply-demand unbalance problem, it is very important to research the taxi traffic system and advance its operational level.

The research achievements could be divided into two types of model and they were aggregated models and equilibrium models. The relevant researches of aggregated models were that: Douglas (1972) was the precursor of the first studies. He considered a taxi market where taxi service was the cruising one and the fares were scheduled by management department and the entry is free [1]. The relevant researches of equilibrium models based on the network structure were that: Yang and Wong presented a series of models during the years 1997-2011 studying the taxi market in the network of Hong Kong [2].

At present, the literatures published are almost based on a single taxi service mode, either cruising mode or dispatch mode. No models have integrated them at the same time. However, with the advancement of taxis installed GPS, the application of dispatch mode becomes more and more frequently so that the integrated service mode considering the existence of cruising and dispatch modes will be the future development trend.

Based on this, this paper is structured as the followed chapters: the second chapter analyzes the characteristic of the integrated taxi service mode and establishes the probability model of trip mode choice for traveler. The third chapter describes the supply-demand equilibrium state with the elastic customer demand in the taxi market and constructs a equilibrium model of taxi on network. The 
next chapter presents the relationship function between the passage waiting time and taxi search/waiting time. The fifth chapter gives the detailed steps of the algorithm to solve taxi model. Finally, the last chapter contains the conclusion and proposes the future extension in taxi field.

\section{The trip mode choice model}

Suppose that there are the cruising service mode and the dispatch one existing in the taxi market at the same time. If the total number of taxis on network is $N u m$ and the proposition of taxis installed GPS is $\alpha$, the number of taxis for the cruising service is $N u m *(1-\alpha)$ and the number of taxis installed GPS is $N u m^{*} \alpha$. The taxis installed GPS can provide not only the dispatch service but also the cruising service.

Suppose that there are two taxi service modes and there is other public transit to be alternative mode chosen by customers on network at the same time.

According to the trip mode choice of travelers, the probability that reflects the split rate of cruising taxi service and dispatch taxi service and other transit modes is followed as:

$$
P_{i j}^{r c}=\frac{\exp \left\{-\theta^{\prime} h_{i j}^{r c}\right\}}{\exp \left(-\theta^{\prime} h_{i j}\right)+\sum_{r=1.2} \exp \left(-\theta^{\prime} h_{i j}^{r c}\right)}, i \in I, j \in J
$$

Where ${ }^{P_{i j}{ }^{r c}}$ is the probability to choose a given taxi service mode from zone $i$ to zone $j ; r \in R, R=\{1,2\}, r=1$ is the cruising taxi service chosen by customer, $r=2$ is the dispatch taxi service chosen by customer; $h_{i j}^{1 c}$ is the full travel cost of the customer who chooses the cruising taxi service from zone ${ }^{i}$ to zone ${ }^{j}{ }^{h_{i j}^{2 c}}$ is the full travel cost of the customer who chooses the dispatch taxi service from zone ${ }^{i}$ to zone ${ }^{j}{ }^{h_{i j}}$ is the full travel cost of the customer who chooses the other transit modes from zone ${ }^{i}$ to zone ${ }^{j}$, and the structure of ${ }^{h_{i j}}$ used here is from[3]; the value of parameter $\theta^{\prime}$ reflects the degree of uncertainty in customer demand and taxi services from the perspective of customers.

Here, the full travel cost function of customer who chooses the cruising taxi service is followed as:

$$
h_{i j}^{1 c}=F_{0}+\pi t_{i j}+\lambda_{1} t_{i j}+\lambda_{2} w^{1 c}
$$

Where $F_{0}$ is the taxi flag-drop charge and $\pi_{\text {is }}$ the variable charge per unit ride time; ${ }_{i j}$ is the travel time via the shortest route from origin zone ${ }^{i}$ to destination zone ${ }^{j} ; F_{0}+\pi t_{i j}$ is the fare of a taxi ride from zone ${ }^{i}$ to zone ${ }^{j} ; \lambda_{1}$ and ${ }^{\lambda}$ are the customers' monetary values of unit in-vehicle time and waiting time respectively; $w^{1 c}$ is the waiting time of customer who chooses the cruising service; $\lambda_{1} t_{i j}$ and $\lambda_{2} w^{1 c}$ are the in-vehicle travel cost and waiting cost for the cruising taxi service respectively.

Here, the full travel cost function of customer who chooses the dispatch taxi service is followed as:

$$
h_{i j}^{2 c}=F_{0}+\pi t_{i j}+\lambda_{1} t_{i j}+\lambda_{2} w^{2 c}+u
$$


Where $F_{0}+\pi t_{i j}$ is the taxi ride fare of dispatch taxi service and the same as the one of cruising service; $\lambda_{1}$ and $\lambda_{2}$ are the same as the above explanation; $w^{2 c}$ is the waiting time of customer who chooses the dispatch service; $u$ is the communication cost which is used to dial to book the dispatch taxi service.

Therefore, the total customer demand choosing cruising service is given by

$$
\sum_{i \in I} \sum_{j \in J} Q_{i j}^{1 c}=\sum_{i \in I} \sum_{j \in J} Q_{i j}^{c} P_{i j}^{1 c}
$$

Where ${ }^{c}{ }^{c}$ is the total customer demand on network.

At the same time, the total customer demand choosing dispatch service is given by

$$
\sum_{i \in I} \sum_{j \in J} Q_{i j}^{2 c}=\sum_{i \in I} \sum_{j \in J} Q_{i j}^{c} P_{i j}^{2 c}
$$

\section{The equilibrium model of taxi on network}

(1) Introduction of model variables

Assume that there is a road network $G(V, A)$, where $V$ is the set of nodes and $A$ is the set of links on network. Suppose that the demand-supply equilibrium exists on network. In any given hour, the number of customers who choose the taxi travel from origin zone $i$ to destination zone $j$ is $\sum_{i \in I} \sum_{j \in J} Q_{i j}^{1 c}+\sum_{i \in I} \sum_{j \in J} Q_{i j}^{2 c} \quad($ person $/ \mathrm{h})$ and the customer demand is elastic. $I$ and $J$ are the sets of customer origin and destination zones respectively. $t_{a}\left(v_{a}\right)$ is the travel time on link $a \in A$. The total traffic flow $v_{a}$ includes both the number of taxis and normal traffic volume. ${ }^{k}{ }_{i j}^{k}$ is the travel time on route $k \in R_{i j}, t_{i j}^{k}=\sum_{a \in A} t_{a}\left(v_{a}\right) \delta_{i j}^{a k}$,where $R_{i j}$ is the set of routes between O-D pair $(i, j)$ and $\delta_{i j}^{a k}=1$ if route $k$ uses link $a$ and 0 otherwise. ${ }_{i j}=\min \left(t_{i j}^{k}, k \in R_{i j}\right)$. The time fraction is an hour, and congestion on network isn't considered here, and a trip is that a taxi is only occupied by a customer.

(2) Taxi service time relationship

Suppose that there are $N^{1}$ cruising taxis traveling on network and consider one unit period (1h). The customer demand is obtained by the above trip mode choice model. The total taxi service time include the occupied time and the unoccupied time. The total occupied time $T^{o t}(\mathrm{veh} \cdot \mathrm{h})$ is the taxi-hours required to complete all cruising trips for all taxis and is given by:

$$
T^{o t}=\sum_{i \in I} \sum_{j \in J} T_{i j}^{o t} t_{i j}
$$

Where $T_{i j}^{o t}$ is the occupied taxi movement (veh/h) from zone ${ }^{i \in I}$ to zone ${ }^{j \in J}$ along the shortest route; ${ }^{t}{ }^{i j}$ is explained in Eq. 2 .

The total unoccupied time $T^{v t}(\mathrm{veh} \cdot \mathrm{h})$ consists of the search times of vacant taxis from zone to zone and waiting times within zones and is followed as:

$$
T^{v t}=\sum_{i \in I} \sum_{j \in J} T_{j i}^{v t}\left(t_{j i}+w_{i}^{t}\right)
$$

Where $^{T_{j i}^{v t}}$ is the vacant taxi movement $(\mathrm{veh} / \mathrm{h})$ from zone ${ }^{j \in J}$ to zone $i \in I$ along the shortest route; $w_{i}^{t}$ is the taxi search/waiting time within zone $i \in I ; t_{i j}$ is explained in Eq.2. 
Therefore, the relationship between the total taxi service time and the total number of taxis in terms of the 1 -h period is given by:

$$
\sum_{i \in I} \sum_{j \in J} T_{i j}^{o t} t_{i j}+\sum_{j \in J} \sum_{i \in I} T_{j i}^{v t}\left(t_{j i}+w_{i}^{t}\right)=N^{1} * 1
$$

Where $N^{1}$ is the total number of cruising taxi (veh).

(3)Route choice behavior of taxi drivers

Suppose that once a customer ride is completed at a destination zone $j \in J$, the taxi driver could either stay in the same zone or move to other zones to find a next customer. Based on this, each driver tries to minimize his expected search time to meet the next customer, and the expected search time is a random variable due to variations in perceptions and the random arrival of customers. This random variable is assumed to be identically distributed with a Gumbel density function $[4,5]$. Thus the probability that a vacant taxis meeting a customer eventually in zone $i \in I$ is given by

$$
P_{i / j}=\frac{\exp \left\{-\theta^{\prime \prime}\left(t_{j i}+w_{i}^{t}\right)\right\}}{\sum_{m \in I} \exp \left\{-\theta^{\prime \prime}\left(t_{j m}+w_{m}^{t}\right)\right\}} \underset{i \in I j \in J}{ }
$$

Where ${ }^{P^{i} / j}$ is the probability of taxi that searches and meets the next customer in zone $i \in I$ after taking a customer to zone ${ }^{j \in J} ; \theta^{\prime \prime}$ is a nonnegative parameter and its value reflects the degree of uncertainty on customer demand and taxi services from the perspective of individual taxi drivers, which is explained in [6].

(4)The supply-demand equilibrium relationship

In a stationary equilibrium state, in terms of cruising taxi service, the movements of vacant taxis on network should meet the customer demands at all origin zones, or every customer will eventually receive taxi service after waiting. In the equilibrium state, the supply-demand relationship of vacant taxi for cruising service is followed as:

$$
\begin{aligned}
& \sum_{i \in I} T_{j i}^{v t}=\sum_{i \in I} Q_{i j}^{1 c}=Q_{j}^{1 c}, j \in J \\
& \sum_{j \in J} T_{j i}^{v t}=\sum_{j \in J} Q_{j}^{1 c} P_{i / j}=Q_{i}^{1 c}, i \in I
\end{aligned}
$$

In the equilibrium state, the supply-demand relationship of occupied taxi for cruising service is followed as:

$$
\sum_{i \in I} \sum_{j \in J} T_{i j}^{o t} t_{i j}=\sum_{i \in I} \sum_{j \in J} Q_{i j}^{1 c} t_{i j}
$$

In the equilibrium state, the supply-demand relationship for dispatch service is followed as:

$$
N^{2}=\sum_{i \in I} \sum_{j \in J} Q_{i j}^{2 c}=\sum_{i \in I} \sum_{j \in J} Q_{i j}^{c} P_{i j}^{2 c}
$$

Where $N^{2}$ is the number of taxis which provide dispatch service.

Therefore, the total number of taxis which provides cruising service is given by:

$$
N^{1}=N u m-N^{2}
$$

(5)A combined trip distribution and assignment UE model with elastic demand

As far as the cruising taxi service is concerned, the taxi movement on network is described by using a combined trip distribution and assignment UE model with elastic demand ${ }^{l i c}$. This mathematical programming model is given by: 


$$
\begin{gathered}
\min Z=\sum_{a \in A} \int_{0}^{v_{a}} t_{a}(w) d w+\frac{1}{\theta^{\prime \prime}} \sum_{j \in J} \sum_{i \in I} T_{j i}^{v t}\left(\ln T_{j i}^{v t}-1\right)-\sum_{i \in I} \sum_{j \in J} \int_{0}^{Q_{i j}^{l c}} Q_{i j}^{-1}(w) d w \\
\text { subject } \quad t o: \sum_{i \in I} T_{j i}^{v t}=\sum_{i} Q_{i j}^{1 c} \\
\sum_{j \in J} T_{j i}^{v t}=\sum_{j} Q_{i j}^{1 c} \\
\sum_{k \in R_{i j}} f_{i j}^{k}=T_{i j}^{n}+T_{i j}^{o t}+T_{i j}^{v t} \\
v_{a}=\sum_{i \in I} \sum_{j \in J} \sum_{k \in R_{i j}} \delta_{i j}^{a k} f_{i j}^{k} \\
f_{i j}^{k} \geq 0 \\
T_{j i}^{v t}>0
\end{gathered}
$$

Where ${ }^{T_{j i}^{v t}}$ is explained by Eq.(7); ${ }^{T_{i j}^{o t}}$ is explained by Eq.(6); ${ }^{T_{i j}^{n t}}$ is normal traffic movements from origin zone $i \in I$ to destination zone $j \in J$ (veh/h); $f_{i j}^{k}$ is traffic flow on route $k$ from zone $i \in I$ to zone $^{j \in J} ; Q_{i j}^{-1}$ is the inverse function of demand function.

(6)Taxi search/waiting time

Based on the calculated $T_{j i}^{v t}$ and the completed iterative balancing factors $A_{i}$ and ${ }^{B_{j}}$ form [6], taxi search/waiting time is obtained by using Eq.16 and Eq.17. The detailed deduction of Eq.16 and Eq. 17 is from [3]. They are necessarily improved here and are given by:

$$
f=\exp \left\{\frac{-\theta^{\prime \prime}\left(N u m-N^{2}-\sum_{i \in I} \sum_{j \in J} Q_{i j}^{1 c} t_{i j}-\sum_{i \in I} \sum_{j \in J} T_{j i}^{v t} t_{j i}\right)-\sum_{i \in I} \sum_{j \in J} Q_{i j}^{1 c} \ln A_{i}}{\sum_{i \in I} \sum_{j \in J} Q_{i j}^{1 c}}\right\}
$$

Taxi waiting times can then be obtained as

$$
w_{i}^{t}=-\frac{\ln \left(f A_{i}\right)}{\theta^{\prime \prime}}
$$

\section{Customer waiting time at demand-supply equilibrium state}

According to [3], the function relationship between the passage waiting time and taxi search/waiting time is obtained by introducing the Cobb-Douglas type of customer-taxi meeting function into the model, and that is given by

$$
W_{i}^{c}=\frac{1}{D_{i} \sum_{j \in J} Q_{i j}^{1 c} w_{i}^{t}}
$$

Where $W_{i}^{c}$ is the passage waiting time (h); $D_{i}$ is the location-dependent constant. If the meeting between customer and taxi occur at fixed taxi stands (point meeting), the corresponding $D_{i}$ is assumed to have a large value, such as $D_{i}=10^{4}(1 / \mathrm{veh} \cdot \mathrm{h})$; if it is the residential zones, $D_{i}$ is 0.01 $(1 / \mathrm{veh} \cdot \mathrm{h})$; if it is the commercial zones, ${ }^{D_{i}}$ is $0.1(1 / \mathrm{veh} \cdot \mathrm{h})$. 


\section{The solution algorithm of equilibrium model}

The general procedure is set out below:

Step 1.Initialization: set an initial set of customer-waiting times, ${ }_{i}^{c(0)}, i \in I$, let $n:=1$.

Step2.Customer demand of different trip modes updating: update $Q_{i j}^{r c(n)}=Q_{i j}^{c} P_{i j}^{r c(n)}, i \in I$, $j \in J, \quad r \in R$, where $P_{i j}^{r c(n)}$ is given by Eq.1.

Step3.A equilibrium model of taxi on network: solve the equilibrium model of taxi on network for given $Q_{i j}^{r c(n)}, i \in I, j \in J, r \in R$ by the iterative balancing method and thus obtain a set of taxi searching/waiting times, ${ }^{t(n)}, i \in I$.

Step4.Updating customer-waiting time: update $W_{i}^{c(n)}, i \in I_{\text {with }} w_{i}^{t(n)}, i \in I$ according to Eq.18.

Step5.Convergence criterion: if convergence is attained according to Eq.19, then stop. Otherwise, let $n:=n+1$ and go to step 2 .

$$
\left|\frac{W_{i}^{c(n)}-W_{i}^{c(n-1)}}{W_{i}^{c(n-1)}}\right| \leq \varepsilon
$$

\section{Conclusions}

This paper established an equilibrium model of taxi service on network. This model reflected the existence of integrated service modes including cruising and dispatch modes and considered the influence of elastic demand distribution. Based on these, in supply-demand equilibrium, a number of performance measures such as taxi search/waiting time and customer waiting time were obtained, which can examine the level of taxi service on network and further provide the meaningful references and theoretical support for advancing the operation level of taxi market. With the proposed equilibrium model of taxi on network, this model could be usefully extended in the regulation policies of taxi market based on the integrated service modes, especially the pricing and the entry number of taxi market.

\section{References}

[1] Douglas G. Price Regulation and optimal service standards. The taxicab industry,1972.

[2] Josep Maria Salanova et.al.A review of the modeling of taxi services[J].Procedia Social and Behavioral Sciences 20 (2011) 150-161.

[3] Yang H., Cowina W.Y.Leung, et al. Equilibria of bilateral taxi-customer searching and meeting on networks[J]. Transportation Research: Part B, 2010, 44: 1067 1083.

[4] Bian Yang, Wan Jian, Lu Jian. equilibrium model of urban taxi service network[J].Journal of traffic and transportation engineering, 2007.2, 7(1):93 98.

[5] Luo Ruigao, Shifeng. A taxi service network equilibrium model with the influenced of demand distribution[J].Journal of railway science and engineering, 2009.2,6(1):87 91.

[6] Yang H.and Wong S.C. A network model of urban taxi services. Transport Research B, 1998,Vol.32,No.4,pp235-246. 